\title{
Fijación externa con sistema de riel de reconstrucción como tratamiento para seudoartrosis del fémur distal
}

\section{External fixation with reconstruction rail system as a treatment for pseudoarthrosis of the distal femur}

\author{
Morales-Avalos R, * Rodríguez-Torres RA, ${ }^{*}$ Martínez-Manautou LE, ${ }^{*}$ \\ Rivera-Zarazúa S, * Requena-Araujo P, * Blázquez-Saldaña J, * Peña-Martínez VM*
}

Hospital Universitario «Dr. José Eleuterio González».

RESUMEN. La seudoartrosis del fémur distal, también llamada «no unión del fémur distal» (NUFD), representa una complicación poco frecuente asociada a una fractura causada por un traumatismo de alta energía; su tratamiento es controversial, ya que existe una gran variedad técnicas quirúrgicas que pueden ser implementadas desde la reducción abierta más la fijación interna con placas hasta el uso de placas de ángulo fijo combinadas con autoinjerto óseo. Exponemos el caso de un hombre de 24 años de edad, el cual se presenta a la consulta con el antecedente de haber sufrido una fractura supracondílea femoral izquierda con un año de evolución, tratada mediante una osteosíntesis del fémur distal con tornillos condilares dinámicos (placa DCS); mostró una evolución tórpida con dolor severo en el tercio distal del muslo izquierdo y limitación funcional, desarrolló una deformidad en varo progresiva del muslo izquierdo que causó un acortamiento de $3.8 \mathrm{~cm}$ de la extremidad. Se identificó un foco de seudoartrosis, el cual se retiró quirúrgicamente, se recanalizó el canal intramedular y se realizó una osteotomía de cuña cerrada lateral para corregir la angulación y permitir la reducción. Se colocaron tornillos de hidroxiapatita de $5.5 \mathrm{~mm}$ (tres proximales y tres distales) y un fijador externo. Además se hizo una revisión de la literatura enfocada en el uso de la fijación externa como tratamiento de la seudoartrosis del fémur distal.
ABSTRACT. Distal femur pseudoarthrosis also called «distal femur non-union» (DFNU) represents a rare complication associated with a fracture caused by highenergy trauma; its treatment is controversial, as there is a wide variety of surgical techniques that can be implemented, from open reduction plus internal fixation with plates, to the use of fixed angle plates combined with bone autograft. We expose the case of a 24-year-old man who shows up for consultation with the history of having presented a left femoral supracondylar fracture with a year of evolution, treated by osteosynthesis of the distal femur with dynamic condylar screws (DCS plate); developed a torpid evolution with severe pain in the distal third of the left thigh and functional limitation, developed a progressive varus deformity of the left thigh that caused a shortening of 3.8 $\mathrm{cm}$ of the limb. A focus of pseudoarthrosis was identified, which was surgically removed, the intramedullary canal was recanalized, and a lateral closed wedge osteotomy was performed to correct the angulation and allow reduction. 5.5 $\mathrm{mm}$ (three proximal and three distal) hydroxyapatite screws and an external fixator were placed. In addition, a review of the literature focused on the use of external fixation was carried out as a treatment for the pseudoarthrosis of the distal femur.

\footnotetext{
* Módulo de Politrauma, Reconstrucción y Fijación Externa. Departamento de Ortopedia y Traumatología, Hospital Universitario «Dr. José Eleuterio González», Universidad Autónoma de Nuevo León, Monterrey, Nuevo León, México.

Correspondencia:

Dr. Rodolfo Morales Avalos

Módulo de Politrauma, Reconstrucción y Fijación Externa, Servicio de Ortopedia y Traumatología

Hospital Universitario «Dr. José Eleuterio González» Universidad Autónoma de Nuevo León (UANL)

Piso 4 del Hospital Universitario, Avenida Francisco I Madero y Dr. Eduardo Aguirre Pequeño, Mitras Centro, C.P. 66460,

Monterrey, Nuevo León, México.

Tel. 818350-7313,

E-mail: rodolfot59@hotmail.com

Citar como: Morales-Avalos R, Rodríguez-Torres RA, Martínez-Manautou LE, Rivera-Zarazúa S, Requena-Araujo P, Blázquez-Saldaña $\mathrm{J}$ et al. Fijación externa con sistema de riel de reconstrucción como tratamiento para seudoartrosis del fémur distal. Acta Ortop Mex. 2020; 34(5): 309-312. https://dx.doi.org/10.35366/97994
} 
Palabras clave: Seudoartrosis, fémur, fijador externo, no unión, tratamiento.
Keywords: Pseudoarthrosis, femur, external fixator, no union, treatment.

\section{Introducción}

Las fracturas del fémur distal representan alrededor de $0.4 \%$ de todas las fracturas y $3 \%$ de las fracturas femorales. ${ }^{1}$ En la mayoría de los casos son causadas por traumatismos de alta energía en personas jóvenes y de baja energía en personas mayores. Las seudoartrosis representan un subconjunto poco frecuente de complicaciones, éstas suceden posterior al tratamiento quirúrgico de fracturas de fémur distal con una incidencia de 5 a $8 \% .{ }^{2}$ Actualmente no existe un consenso formal sobre qué dispositivo de fijación es óptimo para las fracturas asociadas a seudoartrosis del fémur distal. Los fijadores externos se han usado sólo como un manejo temporal para permitir la cicatrización de los tejidos blandos y el cuidado de heridas principalmente en seudoartrosis infectadas y seudoartrosis complejas. ${ }^{2}$ Sin embargo, existen pocos estudios que analizan el uso de la fijación externa como un tratamiento definitivo para la seudoartrosis del fémur distal con resultados aceptables.

Presentamos el caso de un paciente con una seudoartrosis de fémur distal, complicada con acortamiento de la extremidad y deformidad, tratada exitosamente mediante el uso de fijación externa.

\section{Reporte de caso}

Hombre de 24 años de edad se presentó en la consulta con el antecedente de haber sufrido una fractura supracondílea femoral izquierda un año antes de haber acudido. La fractura fue extraarticular de tipo metafisario simple con una línea principal a $9 \mathrm{~cm}$ de la articulación de la rodilla. Como tratamiento se colocó una férula posterior y se realizó cirugía 15 días después en una institución externa, se efectuó una reducción abierta y una osteosíntesis del fémur distal con tornillos condilares dinámicos (placa DCS). Fue dado de alta con la indicación de no apoyar la extremidad afectada y se le dio seguimiento por parte de la consulta para pacientes ambulatorios. El paciente mostró una evolución tórpida que persistía con dolor severo en el tercio distal del muslo izquierdo y limitación funcional, posteriormente desarrolló una deformidad en varo progresiva del muslo izquierdo que causaba un acortamiento de $3.8 \mathrm{~cm}$ de la extremidad, mostraba una marcha disbásica y requería el uso de muletas para caminar. Los rangos de movimiento en la rodilla izquierda fueron de 50 grados de flexión y -20 grados de extensión.

El paciente fue informado sobre la intención de que los datos de su caso se publicaran con fines académicos y dio su aprobación.

\section{Técnica quirúrgica}

Se realizó un abordaje lateral del muslo sobre la herida previa y se disecó de forma roma siguiendo el tejido cicatrizal postquirúrgico. La placa DCS presentaba aflojamiento y fractura de dos tornillos corticales, los cuales no se pudieron retirar (Figura 1). Se retiró el foco de seudoartrosis, se recanalizó el canal intramedular y se realizó una osteotomía de cuña cerrada lateral para corregir la angulación y permitir la reducción. Se colocaron tornillos de hidroxiapatita de 5.5 $\mathrm{mm}$ (tres proximales y tres distales) y fijador Rekrea ${ }^{\circledR}$ (Cittiefe). La cabeza proximal se usó como un poste y la cabeza
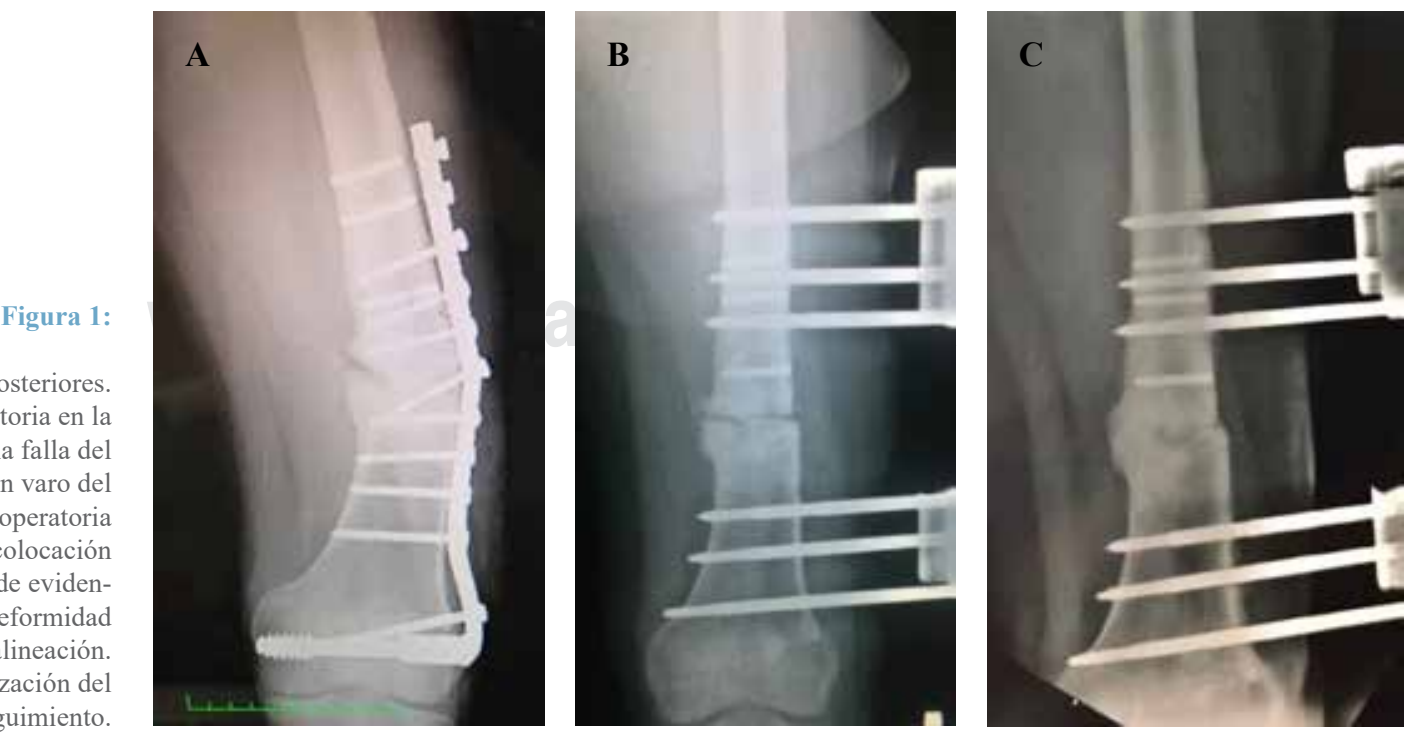

Proyecciones anteroposteriores. A) Radiografía preoperatoria en la que se puede observar la falla de implante y la deformidad en varo del fémur. B) Radiografía postoperatoria inmediata después de la colocación del fijador externo; se puede evidenciar la corrección de la deformidad del fémur y su correcta alineación. C) Radiografía a la finalización de 

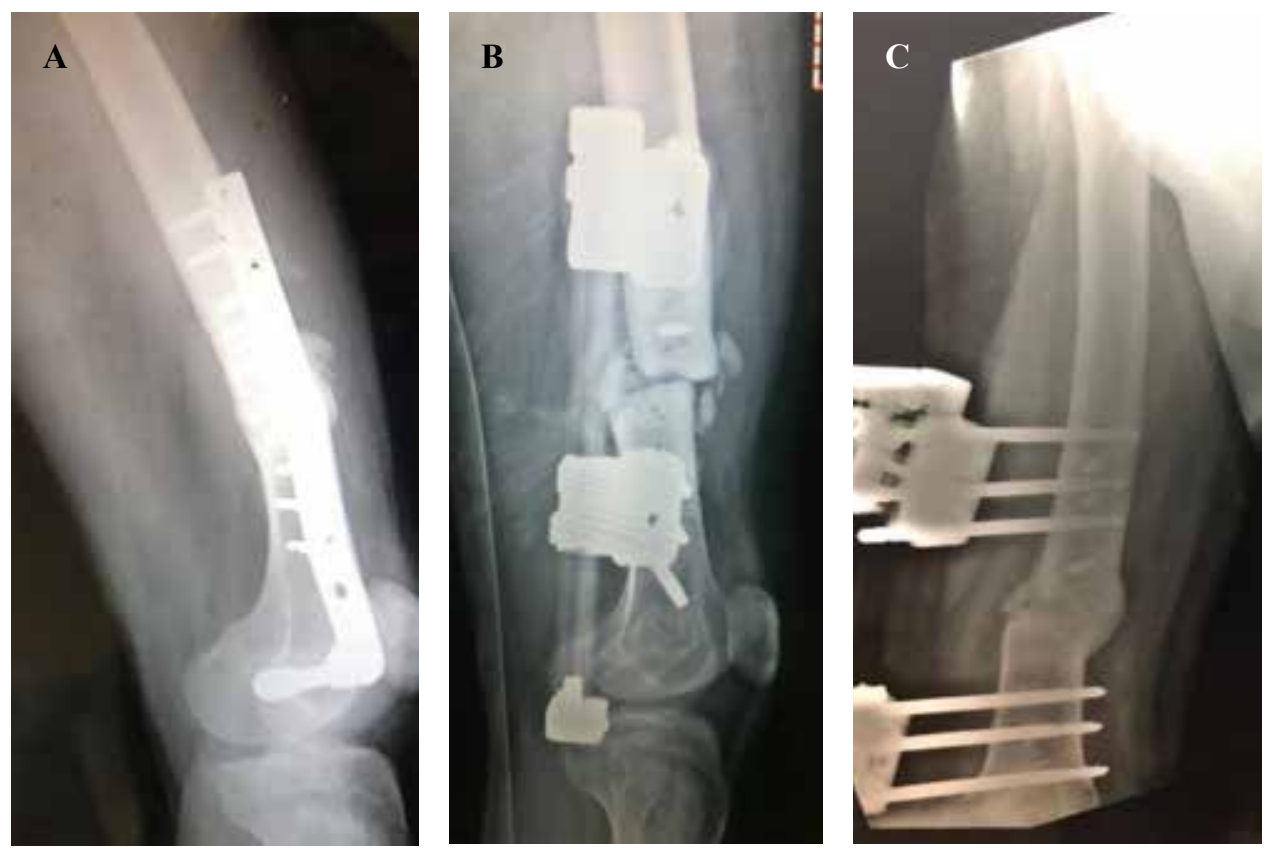

Figura 2:

Proyecciones laterales. A) Radiografía preoperatoria en la que se puede observar la falla del implante y la deformidad en varo del fémur. B) Radiografía postoperatoria inmediata después de la colocación del fijador externo; se puede evidenciar la corrección de la deformidad del fémur y su correcta alineación. C) Radiografía a la finalización del seguimiento.

distal como un compresor / distractor. El sitio de la seudoartrosis se comprimió, se tomaron radiografías de control y la cirugía se cerró por planos hasta la piel (Figura 2).

\section{Evolución}

El paciente fue dado de alta al tercer día. Asistió dos semanas después a la consulta mostrando una flexión de 70 grados y una extensión de -10 grados sin presencia de dolor. En la radiografía de control se observó una buena alineación del fémur y la presencia de formación de callo óseo con una discrepancia en la longitud de las extremidades de $2.80 \mathrm{~cm}$. Actualmente se espera un segundo tiempo quirúrgico para corregir la discrepancia por elongación femoral.

\section{Discusión}

La seudoartrosis del fémur distal, también llamada no unión del fémur distal (NUFD), continúa siendo un problema clínico significativo a pesar de los avances en las técnicas quirúrgicas y las alternativas de fijación. Las cuatro causas principales de NUFD incluyen la estabilidad inadecuada de la fractura, la avascularidad de la región, el patrón de la fractura y el desarrollo de infecciones profundas. ${ }^{3}$ En un estudio multicéntrico de casos y controles de 283 pacientes se estudiaron los factores predictivos de NUFD después de la colocación de una placa lateral bloqueada. Descubrieron que la obesidad, la fractura abierta, la aparición de infección y el uso de acero inoxidable eran los factores de riesgo más importantes de falta de unión y eran independientes de las diferentes tendencias con las que los cirujanos intervinieron en el tratamiento de las seudoartrosis. ${ }^{4}$

En una revisión sistemática realizada por Ebraheim y colaboradores $^{3}$ se encontró que el patrón de fractura más comúnmente asociado a seudoartrosis del fémur distal fue el tipo conminuto metafisario, el tratamiento inicial más frecuente fue la reducción abierta más la fijación interna con placas, el factor de riesgo más importante de desarrollar la no unión fue la presencia del antecedente de fractura abierta y que el tratamiento definitivo más utilizado fue el uso de placas de ángulo fijo combinadas con autoinjerto óseo en las que se logró la unión en $97.4 \%$ de los casos.

Existen problemas específicos relacionados con la NUFD, estos casos por lo general se asocian con un grado significativo de acortamiento de la extremidad y comúnmente ocurren después de múltiples cirugías con una mala cobertura de tejidos blandos y cicatrices importantes. En nuestro caso, el paciente presentó deformidades postraumáticas y acortamiento de la extremidad inferior, en estas situaciones los fijadores externos con un sistema de reconstrucción de rieles pueden ser una buena opción, ya que pueden corregir simultáneamente la longitud y la alineación así como ofrecer mayor estabilización del sitio de la fractura, mejorar la angulación y la rotación. Además, no es necesario alterar los tejidos blandos como con el uso de métodos de fijación interna, en los cuales la disección de los tejidos blandos y la extracción del periostio deterioran aún más el suministro de sangre. La fijación externa se ha descrito en pequeñas series de casos con buenos resultados en la seudoartrosis aséptica. Ali y su equipo ${ }^{5}$ realizaron una revisión retrospectiva de 15 pacientes tratados mediante fijación externa para NUFD, donde el eje y la longitud también se abordaron en un único procedimiento. La unión se logró en 14 de las 15 seudoartrosis; el rango promedio de movimiento antes del tratamiento con el fijador externo fue de $36^{\circ}$; se observaron infecciones menores en los 15 pacientes; sin embargo, no hubo nuevas infecciones profundas como resultado del tratamiento. El reducido número de pacientes en los que se ha 
utilizado esta técnica, indica que se usa con menos frecuencia que la dinamización, el intercambio de clavos y la osteosíntesis de placas. Las complicaciones relacionadas con esta técnica, por ejemplo: osteomielitis, artritis séptica y falla del fijador continúan siendo limitaciones significativas. ${ }^{6} \mathrm{Sin}$ embargo, nuestro paciente no presentó datos de infección ni ninguna otra complicación asociada con el fijador. La dinamización de clavos es otra técnica descrita como una opción para la NUFD; sin embargo, la dinamización tiene una tasa de éxito de $50 \%$ y presenta complicaciones importantes, la más notable es el acortamiento de $>2 \mathrm{~cm}$ que ocurre en $20 \%$ de los pacientes.

En un estudio realizado por Holzman y colaboradores ${ }^{7}$ se utilizaron placas de bloqueo medial con injerto óseo autógeno en 21 pacientes con seudoartrosis del fémur que habían utilizado previamente una placa lateral. Los resultados mostraron que 20 de las 21 seudoartrosis lograron signos radiográficos de unión ósea a los 12 meses, por lo que esta técnica podría ayudar en casos complicados. En otro estudio, Gardner y su equipo ${ }^{8}$ incluyeron como tratamiento la reducción abierta, fijación interna, injerto óseo complementario, colocación de un tornillo de tracción y artrólisis; el número de pacientes estudiados fue de 31, el tiempo promedio de seguimiento fue de 41.5 meses después de la cirugía. Los resultados mostraron que la tasa de unión fue de $97 \%$, aunque se logró un retorno completo al estado funcional previo a la lesión en sólo $84 \%$, el tiempo promedio para la curación fue de 16 semanas. Se han utilizado casi exclusivamente fijadores externos circulares para la NUFM, éstos presentan complicaciones como el uso de varias superficies óseas, lo que implica mayor riesgo de daño a las estructuras vasculares o nerviosas, una gran curva de aprendizaje, alto costo de los implantes y la incomodidad para el paciente al usar el fijador externo. El fijador externo utilizado en el presente estudio es monolateral, lo que implica menor riesgo de daño estructural, una técnica de colocación más sencilla y mayor comodidad para el paciente. Rekrea ${ }^{\circledR}$ (Cittiefe) es un sistema de reconstrucción ósea formado por un cuerpo de fijador monolateral de varias longitudes y por una variedad de cabezas. Está indicado en procedimientos de reconstrucción para el tratamiento del acortamiento de las extremidades superiores e inferiores, la corrección de las deformidades o la pérdida de sustancia ósea con la técnica de alargamiento y transporte óseo en uno o varios niveles.

Se necesitan más estudios de series clínicas para respaldar los resultados de esta terapia a largo plazo. El uso de este implante presenta una curva de aprendizaje intermedia, un costo moderado no demostrado y mayor comodidad para el paciente comparado con los fijadores circulares.

\section{Conclusiones}

El uso de un fijador externo con un sistema de riel de reconstrucción tiene características que lo hacen ideal para el tratamiento de primera línea de la falta de unión del fémur distal.

Referencias

1. Court-Brown CM, Caesar B. Epidemiology of adult fractures: a review. Injury. 2006; 37(8): 691-7.

2. Griffin XL, Parsons N, Zbaeda MM, McArthur J. Interventions for treating fractures of the distal femur in adults. Cochrane Database Syst Rev. 2015; (8): CD010606.

3. Ebraheim NA, Martin A, Sochacki KR, Liu J. Nonunion of distal femoral fractures: a systematic review. Orthop Surg. 2013; 5(1): 46-50.

4. Rodriguez EK, Boulton C, Weaver MJ, Herder LM, Morgan JH, Chacko A, et al. Predictive factors of distal femoral fracture nonunion after lateral locked plating: a retrospective multicenter case-control study of 283 fractures. Injury. 2014; 45(3): 554-9.

5. Ali F, Saleh M. Treatment of distal femoral nonunions by external fixation with simultaneous length and alignment correction. Injury. 2002; 33(2): 127-34

6. Lynch JR, Taitsman LA, Barei DP, Nork SE. Femoral nonunion: risk factors and treatment options. J Am Acad Orthop Surg. 2008; 16(2): 88-97.

7. Holzman MA, Hanus BD, Munz JW, O’Connor DP, Brinker MR. Addition of a medial locking plate to an in situ lateral locking plate results in healing of distal femoral nonunions. Clin Orthop Relat Res. 2016; 474(6): 1498-505.

8. Gardner MJ, Toro-Arbelaez JB, Harrison M, Hierholzer C, Lorich DG, Helfet DL. Open reduction and internal fixation of distal femoral nonunions: long-term functional outcomes following a treatment protocol. J Trauma. 2008; 64(2): 434-8.

Conflicto de intereses: Los autores declaran que no tienen ningún conflicto de intereses.

Financiamiento: Recursos propios del Servicio de Ortopedia de Hospital Universitario de la Universidad Autónoma de Nuevo León (UANL). 